\title{
Assessing response to treatment of exacerbations of bronchiectasis in adults
}

\author{
M.P. Murray, K. Turnbull, S. MacQuarrie and A.T. Hill
}

\begin{abstract}
The present study aimed to assess the effect of intravenous antibiotic therapy on clinical and laboratory end-points in exacerbations of noncystic fibrosis bronchiectasis and to determine whether the outcomes were influenced by the pathogenic organism isolated.

A prospective cohort study was conducted from November 2006 to March 2008 of exacerbations requiring intravenous antibiotics. End-points included 24-h sputum volume, forced expiratory volume in one second (FEV 1 ), forced vital capacity (FVC), incremental shuttle walk test, qualitative sputum microbiology, white cell count, erythrocyte sedimentation rate, C-reactive protein (CRP) and St George's Respiratory Questionnaire (SGRQ). Exacerbations due to Pseudomonas aeruginosa were compared with exacerbations due to other potential pathogenic organisms.

In total, 32 exacerbations were studied. Following 14 days of intravenous antibiotics, all outcomes significantly improved independent of a pathogenic organism, except FEV 1 and FVC. The most responsive markers were: 24 -h sputum volume (reduced in all patients and $80 \%$ had $\geqslant 50 \%$ reduction); sputum bacterial clearance (78.1\%); CRP ( $\geqslant 75 \%$ reduction in $62.5 \%$ ) and SGRQ ( $\geqslant 4$ unit improvement in $89.7 \%$ ). CRP, 24-h sputum volume and SGRQ improved independent of microbial clearance.

In the current study, 24-h sputum volume, microbial clearance, C-reactive protein and St George's Respiratory Questionnaire were the most useful parameters to assess response to treatment of exacerbations of bronchiectasis. Outcomes were similar independent of the pathogenic organism with the exception of forced expiratory volume in one second and forced vital capacity.
\end{abstract}

KEYWORDS: Exacerbations, noncystic fibrosis bronchiectasis, treatment

I nfective exacerbations are a significant cause of morbidity in noncystic fibrosis bronchiectasis and often necessitate utilisation of healthcare resources including in-patient admissions for intravenous antibiotic therapy [1]. The criteria used to define exacerbations requiring antibiotic therapy have been described in chronic obstructive pulmonary disease and include increasing dyspnoea, increasing sputum production and worsening sputum purulence [2]. Similarly, in bronchiectasis, antibiotics are prescribed during exacerbations in patients with increasing cough, increasing sputum volume and worsening sputum purulence. Little is known about how best to assess the effect of antibiotic treatment in the management of such exacerbations. Currently, a successful outcome is only qualitative, relying on the patient's subjective assessment of symptom resolution. Validated, easily accessible and relevant outcome measures are needed [3].

It is not known if the outcome of an exacerbation is influenced by the pathogenic organism. It has been shown that patients who are chronically colonised with Pseudomonas aeruginosa have more severe bronchiectasis, a poorer health-related quality of life (HRQoL) and may have an accelerated decline in forced expiratory volume in one second (FEV1) [4-7].

The aim of the present prospective cohort study was to assess the effect of 2 weeks of i.v. antibiotic therapy for exacerbations of noncystic fibrosis bronchiectasis on a variety of both clinical and laboratory end-points including 24-h sputum volume, FEV1, forced vital capacity (FVC), exercise capacity, systemic inflammation, sputum microbiology and HRQoL. In addition, the study aimed to determine whether these outcomes were influenced by the pathogenic organism isolated.

\section{METHODS}

The current study was a prospective cohort conducted from November 2006 to March 2008 of adults with infective exacerbations of noncystic fibrosis bronchiectasis requiring i.v. antibiotics. Ethical approval was obtained from the Lothian Research Ethics Committee (Edinburgh, UK).

\section{AFFILIATIONS}

Dept of Respiratory Medicine, Royal Infirmary of Edinburgh, Edinburgh, UK.

CORRESPONDENCE

M.P. Murray

Dept of Respiratory Medicine

51 Little France Crescent

Old Dalkeith Road

Edinburgh

EH16 4SA

UK

Fax: 441312421870

E-mail: maevemurray@hotmail.com

Received:

August 082008

Accepted after revision:

September 232008

SUPPORT STATEMENT

M.P. Murray is funded by the Chief

Scientist Office, Edinburgh, UK.

STATEMENT OF INTEREST

None declared. 
An exacerbation was defined as having clinical deterioration with all of the following symptoms: increasing cough; increasing sputum volume; and worsening sputum purulence. Criteria for i.v. antibiotic therapy included failure of oral antibiotics, culture of pathogenic organisms sensitive only to i.v. agents or severe exacerbations necessitating acute inpatient admission.

Exclusion criteria were: cystic fibrosis; active tuberculosis; active allergic bronchopulmonary aspergillosis; active sarcoid; patients on long-term antibiotic therapy (oral or nebulised); and patients with mixed normal flora isolated from their sputum at the start of the exacerbation.

All patients received 14 days of i.v. antibiotic therapy; the antibiotics chosen were based on sputum microbiology prior to commencing treatment or, if necessary, previous sputum microbiology history. All patients were advised to continue chest physiotherapy. Any patient on long-term oral corticosteroid therapy had their dose doubled for the first 7 days of the exacerbation. No other adjunctive treatments were used to manage the exacerbation.

The following clinical and laboratory outcome measures were assessed immediately prior to commencing antibiotic therapy (day 0 ) and on the completion of antibiotics (day 14).

\section{Clinical assessments}

Patients collected their sputum over a $24-\mathrm{h}$ period in clear sterile pots (maximum volume of $45 \mathrm{~mL} \cdot$ pot $^{-1}$ ) and the total volume was measured. No microbiological analysis was performed on this sample.

FEV1 and FVC were recorded to measure lung function. The FEV1/FVC ratio was calculated. A clinically significant response was $>12 \%$ and $>200 \mathrm{~mL}$ improvement from the start of exacerbation [8].

An externally paced, 10-m incremental field walking test was used to measure exercise capacity [9].

\section{Laboratory assessments}

Qualitative sputum bacteriology was performed on a fresh, early morning sputum sample.

Systemic inflammation was measured by white cell count (WCC), erythrocyte sedimentation rate (ESR) and C-reactive protein (CRP).

\section{HRQoL assessment}

The St George's Respiratory Questionnaire (SGRQ) was completed at the start of the exacerbation and 1 week following antibiotic completion [10]. The questionnaire was adapted for the end of exacerbation assessment to ask about symptoms in the preceding week (i.e. the week following antibiotic completion).

The SGRQ is a 50 item self-administered HRQoL questionnaire consisting of three components: symptoms (eight items); activity (16 items); and impacts (26 items). It has previously been validated to reflect impaired HRQoL in bronchiectasis patients [10]. The total score ranges from 0-100; a higher score indicates a poorer HRQoL. A 4-unit difference in the total
SGRQ score has been established as a clinically significant change [10].

\section{Statistics}

The data was normally distributed and are presented as mean \pm SD. For comparing means, a paired t-test was used. Patients that isolated $P$. aeruginosa were compared with those that isolated other potential pathogenic microorganisms (PPMs) at the start of the exacerbation. Fisher's exact test was used to compare groups. A two tailed p-value of $<0.05$ was considered to be statistically significant. Additional subanalysis was conducted to ensure that the reason for needing i.v. therapy or patients who went on to experience multiple exacerbations did not influence the results.

\section{RESULTS}

\section{Patients}

There were 58 exacerbations of noncystic fibrosis bronchiectasis managed with 2 weeks of i.v. antibiotic therapy during the study period. In total, 13 patients had more than one exacerbation during the study period, but only the first exacerbation for each patient was used. Five patients were excluded as mixed normal flora was isolated at the start of the exacerbation and one patient was excluded because they had mucoid sputum at presentation. The final analysis was conducted in 32 individual patients.

Table 1 details baseline patient characteristics, including parameters of disease severity and table 2 provides details of the aetiology of the bronchiectasis. All patients had advanced bronchiectasis with $29(90.6 \%)$ patients being chronically colonised with pathogenic bacteria in the sputum when clinically stable $(65.6 \%$ had $P$. aeruginosa) and having experienced multiple exacerbations in the past year (table 1). Overall,

\begin{tabular}{lc}
\hline TABLE 1 Patient characteristics & \\
\hline Subjects n & 32 \\
Male & $10(31.3)$ \\
Age yrs & $62.8 \pm 9.8$ \\
Nonsmokers & $23(71.9)$ \\
Ex-smokers & $9(28.1)$ \\
Ex-smoker pack-yr history & $34.5 \pm 17.2$ \\
COPD & $3(9.4)$ \\
Asthma & $18(56.3)$ \\
Inhaled corticosteroid therapy & $28(87.5)$ \\
Nebulised bronchodilator therapy & $7(21.9)$ \\
FEV 1 L & $1.57 \pm 0.58$ \\
FEV 1 \% predicted & $66.5 \pm 27.3$ \\
FVC L & $2.49 \pm 0.78$ \\
FVC \% predicted & $85.3 \pm 33.2$ \\
Infective exacerbations in preceding year & $7.4 \pm 6.0$ \\
Number of lobes involved on HRCT & $4.1 \pm 1.7$ \\
Cystic bronchiectasis & $10(31.2)$ \\
Chronically colonised & $29(90.6)$
\end{tabular}

Data are expressed as $\mathrm{n}(\%)$ or mean $\pm \mathrm{SD}$, unless otherwise stated. COPD chronic obstructive pulmonary disease; FEV1: forced expiratory volume in one second; FVC: forced vital capacity; HRCT: high-resolution computed tomography. 
$62.5 \%$ needed i.v. antibiotics because of failure of oral antibiotics, $34.4 \%$ had severe exacerbations necessitating acute in-patient admission and $3.1 \%$ required i.v. antibiotics as the pathogenic organisms isolated were sensitive only to agents available as i.v. preparations.

\section{Sputum bacteriology}

At the start of the exacerbation $P$. aeruginosa was isolated in 19 patients; 10 had nonmucoid and nine had mucoid $P$. aeruginosa. In total, 13 patients isolated other PPMs which included Haemophilus influenzae $(n=4)$, Streptococcus pneumoniae $(n=3)$, Staphylococcus aureus $(\mathrm{n}=2)$, Moraxella catarrhalis $(\mathrm{n}=2)$, Escherichia coli $(\mathrm{n}=1)$ and Serratia species $(\mathrm{n}=1)$.

\section{Antibiotic therapy}

All patients completed 14 days of antibiotic therapy and doses used were as recommended for bronchiectasis/cystic fibrosis in the British National Formula [11]. In the P. aeruginosa group, 13 patients received i.v. ceftazidime ( $2 \mathrm{~g}$ three times daily) and gentamicin (dose per ideal body weight and creatinine clearance); two patients with a previous history of gentamicin toxicity received i.v. ceftazidime ( $2 \mathrm{~g}$ three times daily) and colistin (2 MU three times daily) and three patients with known impaired renal function received i.v. ceftazidime $(2 \mathrm{~g}$ twice daily) and oral ciprofloxacin (500 mg twice daily). The remaining patient who had a known intolerance of ceftazidime received i.v. piperacillin with tazobactam ( $4.5 \mathrm{~g}$ three times daily) and oral ciprofloxacin (500 $\mathrm{mg}$ twice daily).

In the patients with other PPMs isolated, those with isolated $H$. influenzae, S. pneumoniae and $M$. catarrhalis all received the single agent ceftriaxone ( $2 \mathrm{~g}$ once daily). The two patients with isolated S. aureus (one methicillin-sensitive S. aureus and one methicillin-resistant $S$. aureus) received i.v. flucoxacillin (500 mg four times daily) and vancomycin (dose per ideal body weight and creatinine clearance), respectively. The remaining two patients had isolated Serratia spp. and E. coli and both were managed with i.v. ceftazidime ( $2 \mathrm{~g}$ three times daily) and gentamicin (dose per ideal body weight and creatinine clearance).

\section{Corticosteroids}

In total, four patients were receiving long-term oral corticosteroid therapy and had their dose doubled for 7 days. Otherwise no patients received oral corticosteroid therapy.

\begin{tabular}{lc} 
TABLE 2 Aetiology of bronchiectasis \\
\hline $\begin{array}{l}\text { Inactive allergic bronchopulmonary aspergillosis } \\
\text { Burnt out sarcoidosis }\end{array}$ \\
$\begin{array}{l}\text { Post infective (pneumonia, tuberculosis, pertussis, } \\
\quad \text { measles) }\end{array}$ \\
$\begin{array}{l}\text { Ig deficiency (all IgG subclass 2) } \\
\text { Ciliary dyskinesia } \\
\text { Idiopathic }\end{array}$ & $4(31.1)$ \\
\hline
\end{tabular}

Data are presented as $\mathrm{n}(\%)$. Ig: immunoglobulin.

\section{Side-effects}

No adverse side-effects were reported and no changes to treatment regimes occurred. All patients successfully completed 14 days of treatment.

\section{Outcomes: clinical assessments}

24-h sputum volume

Following 14 days of antibiotics the 24-h sputum volume of all patients reduced with a mean reduction of $21.8 \pm 19.9 \mathrm{~mL}$ $(\mathrm{p}<0.0001$; table 3).

All patients had a reduction in their 24 -h sputum volume: $5 \%$ had $<25 \%$ improvement, $15 \%$ had $25-49 \%$ reduction and $80 \%$ had $\geqslant 50 \%$ reduction (fig. 1 ).

\section{Pulmonary function tests}

The FEV1 did not significantly improve, with a mean \pm SD increase of $0.07 \pm 0.2 \mathrm{~L}(5.4 \pm 12.5 \%$; $\mathrm{p}=0.07$; table 3$)$. The FVC did, however, improve by a mean of $0.09 \pm 0.44 \mathrm{~L}(2.9 \pm 22.2 \%$; $\mathrm{p}=0.01$; table 3 ).

Overall, $29 \%$ of patients did not have any improvement in FEV1, 51.6\% had an improvement of $\leqslant 12 \%$ or $\leqslant 200 \mathrm{~mL}, 19.4 \%$ had an improvement of $>12 \%$ and $>200 \mathrm{~mL}$ (fig. 1).

\section{Exercise capacity}

The distance achieved in the incremental field walking test significantly improved by $58.3 \pm 51.0 \mathrm{~m} \quad(48.9 \pm 54.8 \%$; $\mathrm{p}<0.0001$; table 3), following 2 weeks of antibiotic treatment.

In total, $12 \%$ of patients did not show an improvement in exercise capacity, $28 \%$ had $<25 \%$ improvement, $28 \%$ had $25-$ $49 \%$ improvement and $36 \%$ of patients had an improvement of $\geqslant 50 \%$ (fig. 1).

\section{Outcomes: laboratory assessments}

Systemic inflammation

There was a significant improvement in WCC, ESR and CRP (table 3). WCC reduced by a mean of $3.7 \pm 3.7 \times 10^{9} \cdot \mathrm{L}^{-1}$, ESR reduced by a mean of $17.7 \pm 20.9 \mathrm{~mm} \cdot \mathrm{h}^{-1}$ and CRP reduced by a mean of $59.5 \pm 69.6 \mathrm{mg} \cdot \mathrm{L}^{-1}$ (table 3$)$.

WCC did not improve in $9.7 \%$ of patients, $29 \%$ had $50-74 \%$ improvement and no patients had a $\geqslant 75 \%$ improvement in WCC (fig. 1). ESR did not improve in $30 \%$ of patients, $40 \%$ had a $50-74 \%$ improvement and $7 \%$ had a $\geqslant 75 \%$ improvement in ESR (fig. 1). CRP did not improve in $9.3 \%$ of patients, $15.6 \%$ had a $50-74 \%$ improvement and $62.5 \%$ had a $\geqslant 75 \%$ improvement in CRP (fig. 1).

\section{Sputum microbiology}

Qualitative bacterial clearance was achieved in $78.1 \%$ of patients $(p<0.0001$; fig. 1$)$. All patients infected with other PPMs achieved bacterial clearance. Overall, 12 out of 19 patients infected with $P$. aeruginosa achieved bacterial clearance. Of the seven patients with $P$. aeruginosa who did not achieve bacterial clearance, five of these had a mucoid strain. Although microbial clearance was greater in patients with other PPMs compared with patients infected with $P$. aeruginosa, this difference did not achieve statistical significance $(\mathrm{p}=0.07)$. 


\section{TABLE 3 Outcome measures from start to end of exacerbation}

\begin{tabular}{|c|c|c|c|}
\hline 24-h sputum volume $\mathrm{mL}$ & $30.4 \pm 21.9$ & $8.5 \pm 8.4$ & $<0.0001$ \\
\hline FVC L & $2.35 \pm 0.78$ & $2.5 \pm 0.86$ & 0.01 \\
\hline $\mathrm{FEV}_{1 / \mathrm{FVC}}$ & $61.4 \pm 12.3$ & $60.4 \pm 11.5$ & 0.5 \\
\hline Exercise capacity $m$ & $217.0 \pm 168.0$ & $271 \pm 184.0$ & $<0.0001$ \\
\hline CRP $\mathrm{mg} \cdot \mathrm{L}^{-1}$ & $66.9 \pm 70.7$ & $7.4 \pm 11.2$ & $<0.0001$ \\
\hline
\end{tabular}

Data are presented as mean $\pm \mathrm{SD}$, unless otherwise stated. FEV1: forced expiratory volume in one second; FVC: forced vital capacity; WCC: white cell count; ESR: erythrocyte sedimentation rate; CRP: C-reactive protein. ${ }^{\#}$ : normal range $4-11 \times 10^{9} \cdot \mathrm{L}^{-1}$.

\section{HRQoL}

There was a significant improvement in all of the individual domain scores of the SGRQ following completion of antibiotics (table 4). The total score significantly improved with a mean reduction of $13.8 \pm 12.9$ units ( $\mathrm{p}=0.01$; table 4 ). In total, $7.1 \%$ did not show any improvement in SGRQ score, 3.6\% had an improvement of $<4$ units and $89.3 \%$ had an improvement of $\geqslant 4$ units.

\section{Pathogenic organisms}

The response to treatment was compared between patients with $P$. aeruginosa and other PPMs. The responses were not significantly different with the exception of FEV1 and FVC. Patients infected with $P$. aeruginosa showed no improvement in either FEV1 (value at start of exacerbation was $1.52 \pm 0.61 \mathrm{~L}$ improving to only $1.53 \pm 0.58 \mathrm{~L}$ at the end; $\mathrm{p}=0.9$ ) or in FVC (value at start of exacerbation $2.38 \pm 0.88 \mathrm{~L}$ improving to $2.44 \pm 0.81 \mathrm{~L} ; \mathrm{p}=0.3)$. Those patients infected with other PPMs did, however, have a significant improvement in both
FEV1 (improving from $1.32 \pm 0.48 \mathrm{~L}$ to $1.51 \pm 0.61 \mathrm{~L} ; \mathrm{p}=0.01$ ) and FVC (improving from $2.25 \pm 0.62 \mathrm{~L}$ to $2.58 \pm 0.96 \mathrm{~L}$; $\mathrm{p}=0.02)$.

\section{Failure of microbial clearance}

Despite failure of microbial clearance, there were significant improvements in the three most responsive end-points: 24-h sputum volume (mean \pm SD reduction $13.8 \pm 9.1 \mathrm{~mL} ; \mathrm{p}=0.007$ ), $\mathrm{CRP}$ (mean $\pm \mathrm{SD}$ reduction $61.4 \pm 104.4 \mathrm{mg} \cdot \mathrm{L}^{-1} ; \mathrm{p}=0.03$ ) and SGRQ score (mean \pm SD reduction $14.8 \pm 7.2$ units; $\mathrm{p}=0.004$ ).

\section{Need for i.v. antibiotics}

In total, 11 patients required i.v. antibiotics because of failure of oral antibiotics, one patient due to culture of pathogenic organisms sensitive only to i.v. agents and 20 patients due to severe exacerbations necessitating acute in-patient admission.

At the start of the exacerbation, the need for hospitalisation group had worse exercise capacity $(-196 \pm 59.3 \mathrm{~m} ; \mathrm{p}=0.003)$, higher CRP $\left(49.8 \pm 23.4 \mathrm{mg} \cdot \mathrm{L}^{-1} ; \mathrm{p}=0.04\right)$ and WCC $(2.8 \pm 1.3 \times$

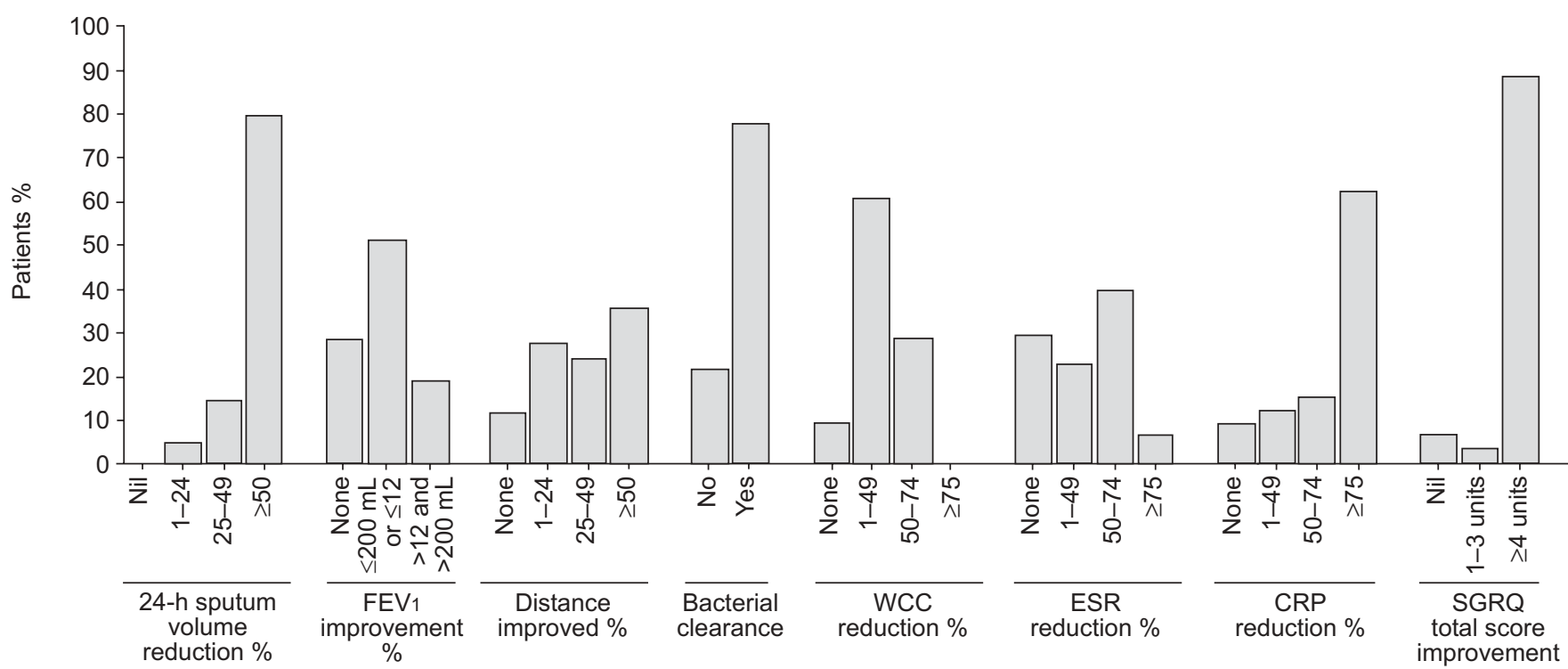

FIGURE 1. Changes in clinical markers following 2 weeks of intravenous antibiotic therapy. FEV1: forced expiratory volume in one second, WCC: white cell count; ESR erythrocyte sedimentation rate; CRP: C-reactive protein; SGRQ: St George's Respiratory Questionnaire. 


\begin{tabular}{llcc} 
TABLE 4 & $\begin{array}{l}\text { Individual domain and total St George's } \\
\text { Respiratory Questionnaire scores from start to } \\
\text { end of exacerbation }\end{array}$ \\
Domain & Start of exacerbation & End of exacerbation & p-value \\
\hline Symptoms & $76.4 \pm 12.9$ & $51.7 \pm 23.1$ & $<0.0001$ \\
Activity & $65.5 \pm 26.4$ & $57.4 \pm 26.5$ & 0.01 \\
Impact & $47.9 \pm 18.4$ & $37.2 \pm 19.2$ & $<0.001$ \\
Total score & $57.9 \pm 17.5$ & $45.7 \pm 20.0$ & $<0.0001$ \\
\hline \multicolumn{4}{l}{} \\
Data are presented as mean $\pm \mathrm{SD}$, unless otherwise stated.
\end{tabular}

$\left.10^{9} \cdot \mathrm{L}^{-1} ; \mathrm{p}=0.04\right)$ and worse SGRQ score (14.4 \pm 5.8 units; $p=0.02)$. There was no significant difference in the other endpoints. Both groups showed a significant improvement in all parameters except in FEV1 and FVC.

\section{Single versus multiple exacerbations}

In total, 19 patients had one exacerbation necessitating i.v. antibiotics and 13 required more than one course of i.v. antibiotics ( $3.6 \pm 1.5$ courses). At the start of the exacerbation there was no difference in the end-points between groups. Patients with a single exacerbation had a significant improvement in all parameters except in FEV1 and FVC. Patients with more than one exacerbation had a significant improvement in all parameters except FEV1.

\section{DISCUSSION}

The present prospective cohort study identified useful clinical and laboratory markers for assessing the response to 2 weeks' i.v. antibiotic treatment in adults with exacerbations of noncystic fibrosis bronchiectasis. Improvements were observed in 24-h sputum volume, sputum bacterial clearance, systemic inflammation, exercise capacity and HRQoL and independent of pathogenic organism. No improvement was observed in FEV1. Microbial clearance, 24-h sputum volume, CRP and SGRQ were the most responsive markers identified.

All patients received 14 days of i.v. antibiotic therapy. The optimum duration of treatment is not known and further trials are needed to define this period but previous studies have administered therapy for between 5 to 28 days [12-15].

All patients with $P$. aeruginosa or Gram-negative organisms were treated with two anti-pseudomonal antibiotics to reduce the chance of developing antibiotic resistance. Those with other PPMs were treated with monotherapy using i.v. ceftriaxone. The optimum therapeutic agents are unknown. The agents used in the current study are known from in vitro sensitivities to be effective agents. The aim of the present study was not to assess the therapeutic regimen but to explore the outcome of 2 weeks of effective antibiotic therapy on a variety of clinical and laboratory parameters.

There are no published international guidelines and no validated methods to allow clinicians to assess response to treatment. Currently, a successful outcome predominantly relies on the subjective assessment of symptom resolution. Thus, objective outcome measures are needed [3]. The present study sought to observe relevant, minimally invasive and easily accessible end-points to assess parameters that may be clinically useful in monitoring response to therapy. Four particularly responsive markers were identified: 24-h sputum volume, qualitative sputum bacteriology, CRP and SGRQ score.

All patients had a significant improvement in 24-h sputum volume, with $80 \%$ having a $\geqslant 50 \%$ reduction. Sputum volume in $24 \mathrm{~h}$ is a potentially useful marker as it is highly pertinent to the condition, noninvasive, easily accessible and inexpensive. It has been used as a marker in previous studies that have assessed potential long-term therapeutic strategies including inhaled steroids and long-term antibiotics [16-21]. A recent study has shown wet weight sputum to be as reliable as dry sputum [22]. The potential limitation of 24-h sputum volume as an outcome measure is the reliance on patient compliance for collection.

Sputum bacteriology as an end-point could involve either complete bacterial clearance or a reduction in bacterial load (colony-forming units $\cdot \mathrm{mL}^{-1}$ ). Direct counts of bacterial load is time consuming, expensive and less likely to be available in the majority of routine (nonresearch) microbiology laboratories. In the current study, $78.1 \%$ had bacterial clearance. In the remaining $22.9 \%$ no information on whether there was a reduction in bacterial load was found as only qualitative cultures were carried out. However, these patients did show improvements in the three other key parameters identified in the present study: 24-h sputum volume, CRP and SGRQ score. Further studies using both qualitative and quantitative cultures would be helpful, although at present, the current findings emphasise the need for more than one end-point to assess response to treatment.

The present authors observed a nine-fold fall in CRP (fall $59.5 \pm 69.6 \mathrm{mg} \cdot \mathrm{L}^{-1}$ ), with $62.5 \%$ having a $\geqslant 75 \%$ improvement following antibiotic therapy. The other markers of systemic inflammation that were observed were WCC and ESR. Although a significant improvement was seen in WCC (1.5fold fall with a fall of $3.7 \pm 3.7 \times 10^{9} \cdot \mathrm{L}^{-1}$ ), the mean WCC at the start of the exacerbation was within normal range and the reduction following antibiotics was small, suggesting WCC is a less relevant marker of treatment response. The mean ESR also improved (1.8-fold fall with a fall of $17.7 \pm 20.9 \mathrm{~mm} \cdot \mathrm{h}^{-1}$ ); however, only $7 \%$ of patients achieved $\geqslant 75 \%$ reduction in ESR. Of these three markers, CRP was most responsive to change.

In total, $89.7 \%$ of patients showed a clinically significant response (i.e. $\geqslant 4$ unit improvement) in SGRQ score. The use of such questionnaires remains predominantly within the research setting as administration and analysis require specific resources and are time consuming.

In keeping with the observed clinical improvements, exercise capacity improved by 1.2 -fold with a rise of $54.1 \pm 51.5 \mathrm{~m}$ and $36 \%$ of patients increasing their distance in the incremental field walking test by $\geqslant 50 \%$. It may be a useful adjunct to other markers.

There was no significant improvement in FEV1 but there was a small 1.06-fold rise in FVC (rise of $144 \pm 306 \mathrm{~mL}$ ). However, there was an improvement seen in FEV1 in patients infected 
with other PPMs, which may reflect the complete bacterial clearance achieved in the current group. A recent review article of exacerbations in bronchiectasis noted FEV1 to be a less sensitive measure in acute exacerbations, particularly in contrast to cystic fibrosis bronchiectasis [3]. Similar findings were made by the authors of a previous study who found that following 2 weeks of treatment with oral amoxicillin there were small improvements in FEV1 (mean improvement $8 \mathrm{~mL}$ ) and FVC (mean improvement $180 \mathrm{~mL}$ ), but these changes were not felt to be clinically useful [23].

In the present study, 24-h sputum volume, microbial clearance, C-reactive protein and St George's Respiratory Questionnaire were the most useful parameters to assess response to treatment of exacerbations of bronchiectasis. Outcomes were similar and independent of the pathogenic organism with the exception of forced expiratory volume in one second and forced vital capacity.

\section{ACKNOWLEDGEMENTS}

The current authors gratefully acknowledge the assistance from J. Pentland and W. Pollock with the incremental shuttle walk test and J. Scott with pharmacy input (all Dept of Respiratory Medicine, Royal Infirmary of Edinburgh, Edinburgh, UK). The present authors would also like to thank P. Jones (St George's University of London, London, UK) for his kind permission to use the St George's Respiratory Questionnaire.

\section{REFERENCES}

1 Kelly MG, Murphy S, Elborn JS. Bronchiectasis in secondary care: a comprehensive profile of a neglected disease. Eur J Intern Med 2003; 14: 488-492.

2 Anthonisen NR, Manfreda J, Warren CP, Hershfield ES, Harding GK, Nelson NA. Antibiotic therapy in exacerbations of chronic obstructive pulmonary disease. Ann Intern Med 1987; 106: 196-204.

3 Chang AB, Bilton D. Exacerbations in cystic fibrosis: 4 - Noncystic fibrosis bronchiectasis. Thorax 2008; 63: 269-276.

4 Martinez-Garcia MA, Soler-Cataluna JJ, Perpina-Tordera M, Roman-Sanchez P, Soriano J. Factors associated with lung function decline in adult patients with stable non-cystic fibrosis bronchiectasis. Chest 2007; 132: 1565-1572.

5 Evans SA, Turner SM, Bosch BJ, Hardy CC, Woodhead MA. Lung function in bronchiectasis: the influence of Pseudomonas aeruginosa. Eur Respir J 1996; 9: 1601-1604.

6 Wilson CB, Jones PW, O'Leary CJ, Hansell DM, Cole PJ, Wilson R. Effect of sputum bacteriology on the quality of life of patients with bronchiectasis. Eur Respir J 1997; 10: 1754-1760.

7 Ho PL, Chan KN, Ip MS, et al. The effect of Pseudomonas aeruginosa infection on clinical parameters in steady-state bronchiectasis. Chest 1998; 114: 1594-1598.
8 Pellegrino R, Viegi G, Brusasco V, et al. Interpretative strategies for lung function tests. Rev Mal Respir 2007; 24: 83-108.

9 Revill SM, Morgan MD, Singh SJ, Williams J, Hardman AE. The endurance shuttle walk: a new field test for the assessment of endurance capacity in chronic obstructive pulmonary disease. Thorax 1999; 54: 213-222.

10 Wilson CB, Jones PW, O'Leary CJ, Cole PJ, Wilson R. Validation of the St. George's Respiratory Questionnaire in bronchiectasis. Am J Respir Crit Care Med 1997; 156: 536-541.

11 British Medical Association and Royal Pharmaceutical Society of Great British National Formulary. 55th Edn. London, Pharmaceutical Press, 2008.

12 Darley ES, Bowker KE, Lovering AM, Harvey JE, Macgowan AP. Use of meropenem $3 \mathrm{~g}$ once daily for outpatient treatment of infective exacerbations of bronchiectasis. J Antimicrob Chemother 2000; 45: 247-250.

13 Chan TH, Ho SS, Lai CK, et al. Comparison of oral ciprofloxacin and amoxycillin in treating infective exacerbations of bronchiectasis in Hong Kong. Chemotherapy 1996; 42: 150-156.

14 Ip M, Shum D, Lauder I, Lam WK, So SY. Effect of antibiotics on sputum inflammatory contents in acute exacerbations of bronchiectasis. Respir Med 1993; 87: 449-454.

15 Tsang KW, Chan WM, Ho PL, Chan K, Lam WK, Ip MS. A comparative study on the efficacy of levofloxacin and ceftazidime in acute exacerbation of bronchiectasis. Eur Respir J 1999; 14: 1206-1209.

16 Tsang KW, Tan KC, Ho PL, et al. Inhaled fluticasone in bronchiectasis: a 12 month study. Thorax 2005; 60: 239-243.

17 Tsang KW, Ho PI, Chan KN, et al. A pilot study of lowdose erythromycin in bronchiectasis. Eur Respir J 1999; 13: 361-364.

18 el-Din MA, Palmer LB, el-Tayeb MN, Khalil I, Gabr MS. Nebulizer therapy with antibiotics in chronic suppurative lung disease. J Aerosol Med 1994; 7: 345-350.

19 Cymbala AA, Edmonds LC, Bauer MA, et al. The diseasemodifying effects of twice-weekly oral azithromycin in patients with bronchiectasis. Treat Respir Med 2005; 4: 117-122.

20 Currie DC, Garbett ND, Chan KL, et al. Double-blind randomized study of prolonged higher-dose oral amoxycillin in purulent bronchiectasis. Q J Med 1990; 76: 799-816.

21 The Medical Research Council. Prolonged antibiotic treatment of severe bronchiectasis; a report by a subcommittee of the Antibiotics Clinical Trials (non-tuberculous) Committee of the Medical Research Council. Br Med J 1957; 2: 255-259.

22 Moran F EJS, Moffitt K, Piper A, Bradley JM. Is sputum load a useful outcome measure for clinical trials in bronchiectasis? Thorax 2006; 61: Suppl. 2, 1-133.

23 Hill SL, Stockley RA. Effect of short and long term antibiotic response on lung function in bronchiectasis. Thorax 1986; 41: 798-800. 